\title{
Drug Delivery to the Eye with a Transdermal Therapeutic System
}

\author{
Akiharu IsowaKi, ${ }^{*}, a$ Akira Ohtori, ${ }^{a}$ Yumi Matsuo, ${ }^{b}$ and Kakuji Tojo ${ }^{b}$ \\ ${ }^{a}$ Kobe Creative Center, Senju Pharmaceutical Co., Ltd.; 1-5-4 Murotani, Nishi-ku, Kobe, Hyogo 651-2241, Japan: and \\ ${ }^{b}$ College of Computer Science and Systems Engineering, Kyushu Institute of Technology; 680-4 Kawazu, Iizuka, Fukuoka \\ 820-8502, Japan. Received June 28, 2002; accepted October 11, 2002
}

\begin{abstract}
A matrix-type transdermal therapeutic system was developed for treating diseases of the eye where it is difficult for drug molecules to reach with conventional topical instillation. Prednisolone was employed as a model drug. An in vivo study using rats showed that the daily application of the patch maintained a constant plasma concentration of the drug, which was equivalent the therapeutic plasma level following three times daily oral administration $(30 \mathrm{mg})$, for approximately $24 \mathrm{~h}$. Transdermal delivery provided equivalent to or higher bioavailability (drug distribution) to the eyeball of topical administration. Moreover, pharmacokinetic analysis indicated that the present transdermal therapeutic system may be clinically effective as a new treatment for ocular diseases.
\end{abstract}

Key words ophthalmic disease; transdermal therapeutic system; ocular bioavailability

Topical instillation is the mainstream the drug therapy for eye disease, especially in anterior eye segments. In general, the bioavailability of drugs administered topically is markedly low because of dilution and excretion by tear fluid, the diffusion barrier of the corneal epithelium, and washout by aqueous flow. ${ }^{1)}$ As a result, only $1 \%$ or less of a dose reaches the aqueous humor and surrounding tissues, and therefore drug molecules applied in the precorneal tear fluid rarely reach the posterior segment tissues such as the vitreous body, choroid, and retina. ${ }^{1)}$ For treating diseases in the posterior segments of the eye, drugs can be directly injected into the vitreous body. However, vitreous injection requires clinical support by ophthalmologists and patients must visit the medical facility frequently. This imposes a heavy physical burden on patients. Ocular medicines may be taken orally, although orally administered drugs are not effective due to first-pass metabolism before reaching the eye tissues.

In this study, we developed a new transdermal delivery system for ocular drugs. The topical drug availability in the eye was investigated following transdermal delivery of prednisolone in rats. Drug bioavailability after transdermal delivery was also compared with that after topical administration.

\section{MATERIALS AND METHODS}

Materials Nine-week-old male Wistar/ST strain rats (Japan SLC Inc., about $300 \mathrm{~g}$ ) and 9-11-week-old female $\mathrm{Hr} / \mathrm{Kud}$ strain hairless mice (Kyudo Co., Ltd., about $30 \mathrm{~g}$ ) were used. Prednisolone was purchased from Nacalai Tesque, Inc. (Tokyo, Japan). Materials used for the transdermal therapeutic system were PE-300 acrylic adhesive (Nippon Carbide Industries Co., Inc.), polyethylene terephthalate film (backing layer and release liner, Lintec Co., Ltd.), isopropyl myristate (IPM, Wako Pure Chemical Industries, Ltd.) as an enhancer, and polyoxyethylated oleyl ether (POE, NOF Corporation) as an enhancer. Other reagents used in the experiment were of special grade.

Preparation of Transdermal Therapeutic System Prednisolone was weighed in a disposable cup and ethyl acetate was added. After dispersing the prednisolone, POE and IPM were added and mixed with a stirrer. The base PE-300 was then added and mixed. After degassing in a vacuum, the mixture was spread over a release liner and ethyl acetate was evaporated at room temperature. After coating with a backing membrane, this membrane was cured in a dryer at about $40^{\circ} \mathrm{C}$ for $8 \mathrm{~h}$ or more. The resultant device was used as the prednisolone transdermal therapeutic system (prednisoloneTTS). The weight fractions of the drug, POE, IPM and PE300 in the device were $3 \%, 20 \%, 20 \%$, and $57 \%$, respectively.

In Vivo Application of Prednisolone-TTS in Rats and Tissue Sampling The abdominal hair of the rats was removed using an electric shaver or a hair clipper, and a cannula was then inserted into the jugular vein for blood sampling. ${ }^{2)}$ After the application of prednisolone-TTS (area, 2 $\mathrm{cm} \times 5 \mathrm{~cm}=10 \mathrm{~cm}^{2} /$ patch; dose, about $3 \mathrm{mg} / \mathrm{patch}$ ) to the shaved central abdominal skin of the rats, about $0.5 \mathrm{ml}$ of blood was collected at 2, 4, 6, 8, 12, 18, and $24 \mathrm{~h}$ from the sampling port in the cannula and plasma was then obtained from the blood by centrifugation. During the experiment, the rats were free moving and allowed free access to food and water. One group of rats was killed separately with an overdose of pentobarbital sodium solution $6 \mathrm{~h}$ after prednisoloneTTS application and their eyeballs were collected to measure the drug concentration in the eye tissues.

Methanol was added to the collected plasma and eyeball samples, then the samples were stirred or finely sectioned, and centrifuged at $3000 \mathrm{rpm}$ for $10 \mathrm{~min}$. The supernatant was collected and evaporated to dryness under reduced pressure. The residue was then dissolved in a mobile phase for column pretreatment and condensation. Prednisolone concentrations in the samples were determined by HPLC. An oral formulation of prednisolone suspended in $1 \%$ carmellose solution was administered $(10 \mathrm{mg} / \mathrm{kg})$ through stomach catheter for comparison. Tissue sampling, pretreatment for protein removal, and extraction were the same as described above.

In Vitro Skin Permeation Experiment Using Hairless Mice The abdominal skin of hairless mice was used for the in vitro skin permeation experiment. Both intact skin and stripped skin, from which the stratum corneum was completely removed by tape stripping, were mounted on an in vitro skin permeation experimental apparatus (Fig. 1). Prednisolone-TTS was applied to the stratum corneum surface and $5 \mathrm{ml}$ of $40 \%$ polyethylene glycol 400 (PEG400) aqueous 


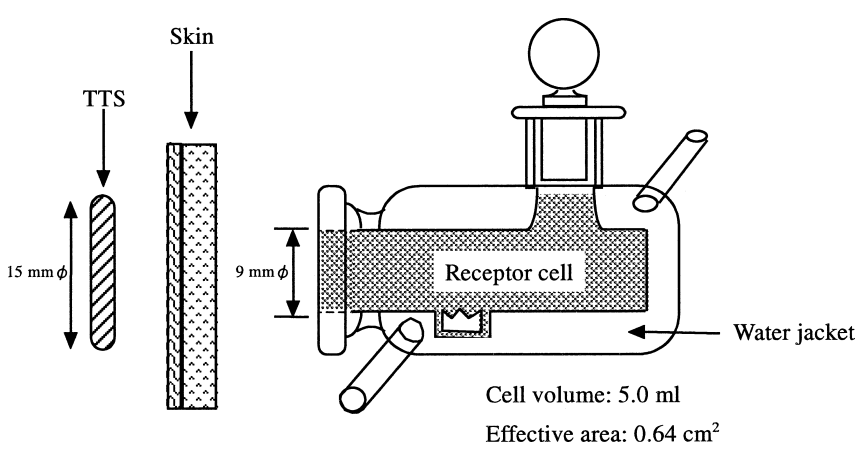

Fig. 1. Experimental Apparatus for in Vitro Skin Permeation Experiment

solution was added to the receptor cell to maintain the sink conditions. The experimental temperature was controlled at $37^{\circ} \mathrm{C}$, and $100 \mu \mathrm{l}$ of the receptor solution was sampled at predetermined time points. Thereafter, the same amount of fresh $40 \%$ PEG400 solution was added to the receptor cell. Skin permeation data were analyzed using the bilayer diffusion/partition model as summarized in a textbook. ${ }^{3)}$ The diffusion coefficient in the stratum corneum $\left(D_{\mathrm{sc}}\right)$, the partition coefficient in the viable skin $\left(D_{\mathrm{vs}}\right)$, the stratum corneum/viable skin partition coefficient $\left(K_{\mathrm{sc}} / K_{\mathrm{vs}}\right)$, and the skin surface drug concentration $\left(C_{\mathrm{s}}\right)$ were calculated from the steady-state permeation rates $(d Q / d t)$ and the time lags $\left(t_{\mathrm{d}}\right)$ in permeation profiles obtained from the intact skin and from the stripped skin in the in vitro experiment. ${ }^{3,4)}$

HPLC Assay of Prednisolone The prednisolone concentration in the in vivo experiment was determined under the following conditions. A semi-microcolumn HPLC system (Nanospace SI-1, Shiseido Co., Ltd.) was used as the HPLC system. Capcell pak MF C-1, 4.0×20 mm (Shiseido), Capcell pak C18 UG80 S5 $\mu \mathrm{m}, 1.5 \times 35 \mathrm{~mm}$ (Shiseido), and Capcell pak C18 UG120 S5 $\mu \mathrm{m}, 1.5 \times 250 \mathrm{~mm}$ (Shiseido) were used for pretreatment, condensation, and analysis of the samples, respectively. The column temperature was $40^{\circ} \mathrm{C}$ and the measuring wavelength was $254 \mathrm{~nm}$ for the analysis. The mobile phases were a mixture of water and acetonitrile (90/10) for pretreatment and condensation, and a mixture of water and acetonitrile (70/30) for analysis.

The prednisolone concentration in the in vitro experiment was determined under the following conditions. The HPLC system used in this experiment comprised a liquid chromatograph (LC-10AS), column oven (CTO-10A), UV-VIS detector (SPD-10A), and system controller (SIL-10A) (all manufactured by Shimadzu Corporation). The column used was a TOSOH TSKgel ODS-80Ts $4.6 \mathrm{~mm} \times 150 \mathrm{~mm}$ (TOSOH Corporation) and its temperature was $45^{\circ} \mathrm{C}$. The measuring wavelength was $254 \mathrm{~nm}$. As the mobile phase, a mixture of methanol and $\mathrm{KH}_{2} \mathrm{PO}_{4} 20 \mathrm{~mm}(60 / 40)$ was used.

Prediction of Clinical Performance (Simulation of Human Plasma Concentration) The human plasma concentration after the application of prednisolone-TTS was estimated using commercially available simulation software for skin pharmacokinetics (SKIN-CAD $\left.{ }^{\mathrm{TM}}\right)^{5}$ ) Parameters of human skin permeation to input the software were difficult to obtain in experiments using human skin, and thus the data obtained from hairless mouse skin were used based on a report that their skin and stratum corneum structure as well as follicular area are pharmacokinetically very similar to those
Table 1. Skin Permeation Parameters of Prednisolone Evaluated in the in Vitro Hairless Mouse Experiment

\begin{tabular}{lrrr}
\hline \hline & Intact skin & & Stripped skin \\
\hline$d Q / d t\left(\mu \mathrm{g} / \mathrm{cm}^{2} / \mathrm{h}\right)$ & $10.96 \pm 0.31$ & & $23.95 \pm 5.64$ \\
$t_{\mathrm{d}}(\mathrm{h})$ & $5.69 \pm 1.02$ & & $0.50 \pm 0.28$ \\
$D_{\text {sc }}\left(\mathrm{cm}^{2} / \mathrm{s}\right)$ & & $1.90 \times 10^{-11}$ & \\
$D_{\text {vs }}\left(\mathrm{cm}^{2} / \mathrm{s}\right)$ & & $1.21 \times 10^{-7}$ & \\
$K_{\text {sc }} / K_{\text {vs }}(-)$ & & $1.49 \times 10^{2}$ & \\
$C_{\mathrm{s}}(\mu \mathrm{g} / \mathrm{ml})$ & & $2.95 \times 10^{5}$ & \\
\hline
\end{tabular}

Each value represents the mean \pm S.D. $(n=3)$. Skin thickness ${ }^{4)}$ : stratum corneum $(\mathrm{sc})=0.0010 \mathrm{~cm}$, viable skin $(\mathrm{vs})=0.0370 \mathrm{~cm}$.

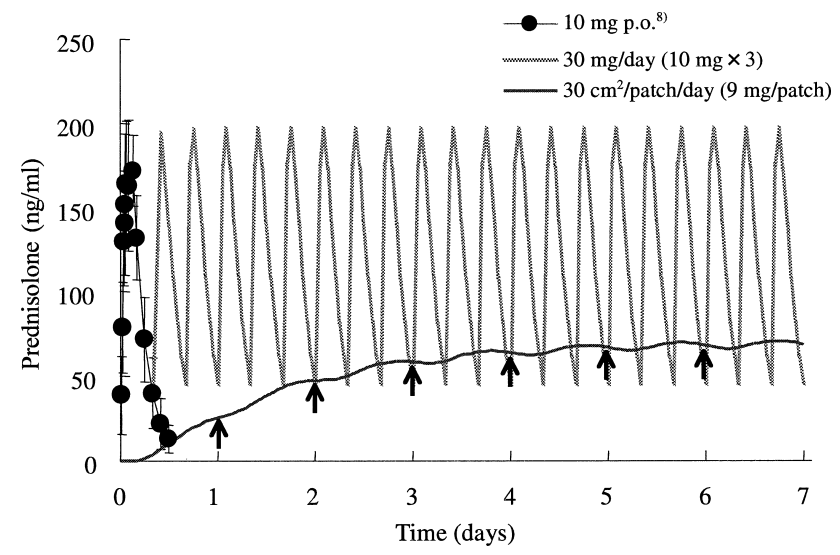

Fig. 2. Predicted Plasma Levels of Prednisolone after Oral Administration and Transdermal Administration in Humans

$\uparrow$, Replaced.

of human skin. ${ }^{6)}$ For the thickness of skin, which greatly influences transdermal absorption, the generally accepted human skin thickness (stratum corneum, $0.002 \mathrm{~cm}$; whole skin, $0.02 \mathrm{~cm}$ ) was employed in the analysis. ${ }^{7)}$ The parameter values used are summarized in Table 1 . The clinical performance was predicted and shown in Fig. 2, where the plasma kinetics after repeated oral administration were also estimated by assuming an oral two-compartment model for comparison. The oral results were estimated using WinNonlin ${ }^{\mathrm{TM}}$ (WinNonlin Version 2.1 Standard, Pharsight Corporation) based on the values cited in the literature. ${ }^{8)}$

\section{RESULTS AND DISCUSSION}

In Vivo Plasma and Eyeball Concentrations of Rat Figure 3 compares the plasma concentration in rats after transdermal administration with that after oral administration. The drug concentration reached the maximum value of $863 \mathrm{ng} / \mathrm{ml}$ at $0.5 \mathrm{~h}$ and quickly decreased with an elimination half-life of $0.7 \mathrm{~h}$ after oral prednisolone administration. This result may indicate that oral administration should be more frequent to maintain an effective plasma concentration. On the other hand, the transdermal application had an initial time lag of about $2 \mathrm{~h}$ after application and a constant high concentration level of drugs was maintained for about $9 \mathrm{~h}$ and then slightly declined up to $24 \mathrm{~h}$ with an elimination half-life of $6 \mathrm{~h}$. Transdermal treatment could therefore maintain a prolonged plasma concentration with one dose. This may justify the efficacy of the transdermal therapeutic system for treating the eye. Additionally, the prednisolone concentration in the 


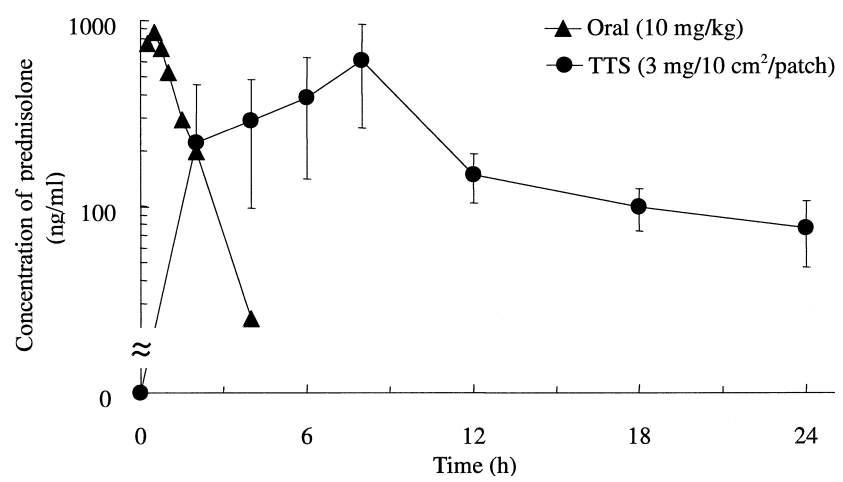

Fig. 3. Plasma Concentration of Prednisolone after oral Administration and Transdermal Administration in Rats

Mean \pm S.D. (oral, $n=2$, TTS, $n=5-16$ ).

Table 2. Eyeball-Plasma Steady-State (6-h) Ratio after Transdermal Administration in Rats

\begin{tabular}{lccc}
\hline \hline & Plasma & Eyeball & $\begin{array}{c}\text { Eyeball/plasma } \\
\text { ratio }\end{array}$ \\
\hline Concentration & $385 \pm 245 \mathrm{ng} / \mathrm{ml}$ & $\begin{array}{c}69.7 \mathrm{ng} / \mathrm{g} \\
\text { tissue } \\
\text { Drug amount }\end{array}$ & $18.1 \%$ \\
\hline $\begin{array}{c}18.1 \mathrm{ng} / 2 \\
\text { eyes }\end{array}$ & $0.8 \%$ \\
\hline
\end{tabular}

Each value represents the mean \pm S.D. [plasma, $n=5-16$; eyeball, $n=1$ (10 eyes)]

eyeball was $18 \%$ of that in plasma at $6 \mathrm{~h}$ during the steadystate absorption process after transdermal application.

Based on the drug concentration in the eyeball and plasma and the tissue volume, fraction of the drug molecules transferred from blood to the eyeballs was evaluated to be $0.8 \%$ under the steady-state at about $6 \mathrm{~h}$ (Table 2). This finding implies that the drug bioavailability in the eyeball was $0.8 \%$ using the present prednisolone-TTS. It was previously reported that ophthalmic solutions generally have drug bioavailability of about $1 \%$ in the aqueous humor through corneal permeation. ${ }^{9)}$ It was also shown that the bioavailability from the aqueous humor to the surrounding tissues, such as the vitreous body and the lens, is 10 -fold lower than that for the aqueous humor. ${ }^{9)}$ Therefore the bioavailability of $0.8 \%$ to the eyeball for the present transdermal therapeutic system is much higher than that with general ophthalmic solutions applied topically.

In Vitro Skin Permeation in Hairless Mice Figure 4 and Table 1 show the in vitro skin permeation profiles and the permeation parameters evaluated from the in vitro experiment, respectively. The diffusion coefficient in the stratum corneum $\left(D_{\mathrm{sc}}\right)$ was the same order of magnitude as that of other drugs with similar molecular weight $(314.5 \leqq \mathrm{M}$.W. $\leqq$ 362.5). ${ }^{4)}$

Prediction of Clinical Performance Based on the skin pharmacokinetic parameters determined above and the body pharmacokinetic parameters calculated from the values cited in the literature ${ }^{8}$ (Table 3), the clinical performance (plasma concentration) of prednisolone-TTS $\left(30 \mathrm{~cm}^{2}=\right.$ about $\left.9 \mathrm{mg}\right)$ was evaluated (Fig. 2). Based on the three times daily oral dose of prednisolone $\left(30 \mathrm{mg} / \mathrm{d}^{10)}\right)$, the concentration profile following oral delivery is also illustrated for comparison.

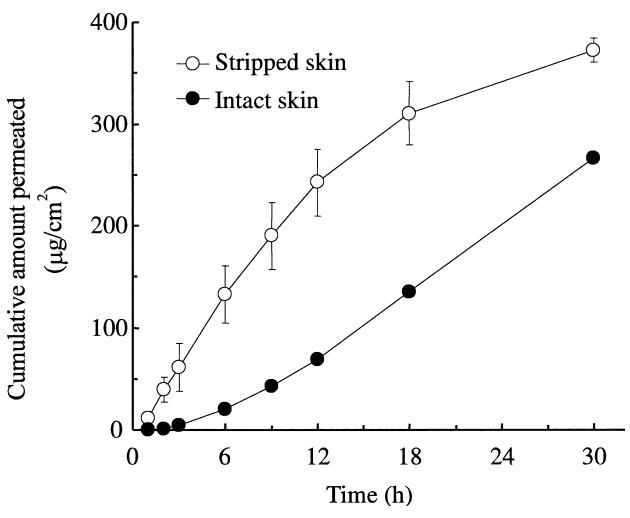

Fig. 4. Time Course of Cumulative Amount of Prednisolone Permeated Across Hairless Mouse Skin for Prednisolone-TTS Containing Enhancer Mean \pm S.D. $(n=6)$

Table 3. Pharmacokinetics Parameters of Prednisolone in Humans

\begin{tabular}{ll}
\hline \hline Elimination rate constant $\left(k_{\mathrm{e}}\right)$ & $9.1 \times 10^{-5} \mathrm{~s}^{-1}$ \\
Transfer rate constant (plasma $\rightarrow$ tissue) $\left(k_{12}\right)$ & $5.3 \times 10^{-4} \mathrm{~s}^{-1}$ \\
Transfer rate constant (tissue $\rightarrow$ plasma) $\left(k_{21}\right)$ & $1.8 \times 10^{-3} \mathrm{~s}^{-1}$ \\
Volume of distribution of central compartment (plasma) $\left(V_{1}\right)$ & $2.3 \times 10^{4} \mathrm{ml}$ \\
Volume of distribution of peripheral compartment (tissue) $\left(V_{2}\right)$ & $6.8 \times 10^{3} \mathrm{ml}$
\end{tabular}

A large fluctuation in the plasma concentration occurred after each oral dosing, while the transdermal therapeutic system showed little fluctuation in plasma concentration at the time of replacement. Since the plasma concentration at $8 \mathrm{~h}$ after repeated oral administration would be the minimum effective concentration, the concentration of about $50 \mathrm{ng} / \mathrm{ml}$ is required for transdermal therapeutic systems. In this prednisolone-TTS, the concentration slowly increased on the first and second days with once daily application of a $30 \mathrm{~cm}^{2}$ patch. After the second day, the effective concentration can be maintained by the repeated application of the present transdermal delivery system.

\section{CONCLUSIONS}

Transdermal delivery of prednisolone molecules to the eye is more effective than conventional topical or oral administration. In a comparison of drug volume between tissues, with the volume and weight of ocular tissue (aqueous humor, lens, vitreous body) and plasma taken into consideration, the bioavailability of $0.8 \%$ in the ocular tissues with transdermal delivery was equal to or higher than that with conventional ophthalmic instillation. Oral preparations of prednisolone result in large peak-trough variations in plasma concentration, which may cause undesirable side effects. On the other hand, the transdermal therapeutic system can provide a nearly constant concentration for an extended period of time.

Based on the ocular bioavailability and the tissue concentration profiles, the transdermal delivery of ocular hydrophobic drugs similar to prednisolone may be safe and effective for treating ocular diseases.

\section{REFERENCES}

1) Tojo K., Ohtori A., J. Eye, 10, 1209-1212 (1993).

2) Bakar S. K., Niazi S., J. Pharm. Sci., 72, 1027-1029 (1983).

3) Martin A., "Physical Pharmacy," fourth edition, Lea \& Febiger, 
Philadelphia, 1993, pp. 538- 545.

4) Tojo K., Chiang C. C., Chien Y. W., J. Pharm. Sci., 76, 123-126 (1987).

5) Mori D., Tojo K., Pharm. Tech. Jpn., 17, 771-778 (2001).

6) Bronaugh R. L., Stewart R. F., Congdon E. R., Toxicol. Appl. Pharmacol., 62, 481-488 (1982).

7) Tojo K., Pharm. Tech. Jpn., 5, 19-27 (1989).
8) Tauber U., Haack D., Nieuweboer B., Kloss G., Vecsei P., Wendt H., Int. J. Clin. Pharmacol. Ther. Toxicol., 22, 48-55 (1984).

9) Tojo K., "The Forefront of Ophthalmology," Frontier Medical Technology Institute, Tokyo, 1999, pp. $64-69$.

10) Gilman A. G., Goodman L. S., Rall T. W., Murad F., "Goodman and Gilman's The Pharmacological Basis of Therapeutics," seventh edition, Macmillan Publishing Company, New York, 1990, p. 1483. 